\title{
A traditional herbal medicine enhances bilirubin clearance by activating the nuclear receptor CAR
}

\author{
Wendong Huang, Jun Zhang, and David D. Moore \\ Department of Molecular and Cellular Biology, Baylor College of Medicine, Houston, Texas, USA
}

\begin{abstract}
Yin Zhi Huang, a decoction of Yin Chin (Artemisia capillaris) and three other herbs, is widely used in Asia to prevent and treat neonatal jaundice. We recently identified the constitutive androstane receptor (CAR, NR1I3) as a key regulator of bilirubin clearance in the liver. Here we show that treatment of WT and humanized CAR transgenic mice with Yin Zhi Huang for 3 days accelerates the clearance of intravenously infused bilirubin. This effect is absent in CAR knockout animals. Expression of bilirubin glucuronyl transferase and other components of the bilirubin metabolism pathway is induced by Yin Zhi Huang treatment of WT mice or mice expressing only human CAR, but not CAR knockout animals. 6,7-Dimethylesculetin, a compound present in Yin Chin, activates CAR in primary hepatocytes from both WT and humanized CAR mice and accelerates bilirubin clearance in vivo. We conclude that CAR mediates the effects of Yin Zhi Huang on bilirubin clearance and that 6,7-dimethylesculetin is an active component of this herbal medicine. CAR is a potential target for the development of new drugs to treat neonatal, genetic, or acquired forms of jaundice.
\end{abstract}

J. Clin. Invest. 113:137-143 (2004). doi:10.1172/JCI200418385.

\section{Introduction}

Jaundice, the accumulation of high levels of bilirubin in the circulation, is particularly common in human neonates (1). Bilirubin is cleared by the liver, and both certain genetic defects and liver diseases can cause hyperbilirubinemia in adults $(2,3)$. Bilirubin is highly hydrophobic, and chronic hyperbilirubinemia results in its deposition in the central nervous system, causing neurotoxicity and encephalopathy. Some evidence suggests that chronic jaundice may also suppress the immune system as well as other normal physiological functions $(4,5)$. Four distinct steps of bilirubin metabolism have been proposed ( 3 , 6). Bilirubin is first imported via the sinusoidal surface of the hepatocyte by solute carrier family 21 , member 6 (SLC21A6; also known as organic anion transporter 2, OATP2) (7). Ligandin, a homodimer or heterodimer of glutathione-S-transferase (GST) A1

Received for publication March 19, 2003, and accepted in revised form October 21, 2003.

Address correspondence to: David D. Moore, Department of Molecular and Cellular Biology, Baylor College of Medicine, One Baylor Plaza, Houston, Texas 77030, USA. Phone (713) 798-3313; Fax (713) 798-3017; E-mail: moore@bcm.tmc.edu.

Conflict of interest: The authors have declared that no conflict of interest exists.

Nonstandard abbreviations used: solute carrier family 21 , member 6 (SLC21A6); glutathione-S-transferase (GST); uridine diphosphate-5'-glucuronosyltransferase (UGT); multidrug resistance-related protein 2 (MRP2); constitutive androstane receptor (CAR); 1,4-bis[2-(3,5-dichloropyridyloxy)]benzene (TCPOBOP); 5 -pregnen-3 $\beta$-ol-20-one-16 $\alpha$-carbonitrile (PCN); 2,3,7,8-tetrachlorodibenzo-p-dioxin (TCDD); cytochrome P450 1A1 (CYP1A1); pregnane $\mathrm{X}$ receptor (PXR); aryl hydrocarbon receptor (AhR). and A2, binds bilirubin with high affinity and thus increases uptake. Bilirubin is then glucuronidated by a specific microsomal bilirubin uridine diphosphate$5^{\prime}$-glucuronosyltransferase (UDP-glucuronosyltransferase 1A1, UGT1A1). The resulting hydrophilic bilirubin diglucuronide is then secreted across the bile-canalicular membrane of the hepatocytes by an active transporter, multidrug resistance-related protein 2 (MRP2) (cMOAT, ABC-C2).

For the last several decades, phototherapy has been used to treat neonatal jaundice. Recently, however, both older and more recent pharmacological approaches to jaundice have been considered (8). Phenobarbital, which induces UGT1A1 activity $(9,10)$ and decreases bilirubin levels in patients (11-13), is the best studied of the older treatments, but there are also numerous remedies for jaundice in various traditional medicines. Yin Zhi Huang and a number of other herbal decoctions containing Yin Chin have been used for centuries in Asia to prevent and treat neonatal jaundice (14). Yin Zhi Huang contains extracts from four different plants: Artemisia capillaris, Gardenia jasminoides Ellis, Rheum officinale Baill, and Scutellaria baicalensis Georgi. Several clinical reports in the Chinese medical literature indicate that Yin Zhi Huang treatment can enhance bilirubin clearance in newborns (15-17). In studies in rats, both Yin Zhi Huang and phenobarbital induced bilirubin glucuronyl transferase and GST activity, and Yin Zhi Huang had a somewhat more potent stimulatory effect on bilirubin clearance than phenobarbital $(18,19)$.

Constitutive androstane receptor (CAR) NR1I3 has been shown to mediate the response of liver to phenobarbital and other "phenobarbital-like" compounds (20-23). Recently, we demonstrated that CAR is a key 
regulator of the bilirubin clearance pathway and that CAR activation increases the rate of bilirubin clearance (24). Here we test the prediction that CAR mediates the effect of Yin Zhi Huang on bilirubin clearance. By screening for CAR activators, we also identify an active compound in Yin Zhi Huang that accelerates this clearance.

\section{Methods}

Yin Zhi Huang and Yin Chin decoction preparations. A Yin Zhi Huang decoction containing four different herbs for gavage was prepared by boiling $40 \mathrm{~g}$ Artemisiae, 14.6 $\mathrm{g}$ Gardenniae, $7.2 \mathrm{~g}$ Rheum, and $12 \mathrm{~g}$ Scutellariae in water for 30 minutes and adjusting the final volume to $40 \mathrm{ml}$. For Yin Chin decoction, only Artemisiae $40 \mathrm{~g}$ was added. Yin Zhi Huang and Yin Chin were obtained from Chinese Herb Inc. (Houston, Texas, USA).

Animal treatment. Mice were hosted in a pathogen-free animal facility under a standard 12 -hour light/12-hour dark cycle. Mice were fed standard rodent chow and water ad libitum. A humanized CAR transgenic mouse line was generated by crossing an albumin promoter/human CAR transgene into the CAR null background, as described previously (25). Different groups of mice appropriately matched in genetic background $(n=3-4)$ were injected i.p. with a solvent (corn oil) or 1,4-bis [2-(3,5-dichloropyridyloxy)]benzene (ТСРОВОР) ( $3 \mathrm{mg} / \mathrm{kg})$, 5 -pregnen-3 $\beta$-ol-20-one-16 $\alpha$ carbonitrile (PCN) $(40 \mathrm{mg} / \mathrm{kg})$, and 2,3,7,8-tetrachlorodibenzo-p-dioxin (TCDD) $(100 \mu \mathrm{g} / \mathrm{kg})$ for 3 days. [4-Chloro-6-(2,3-xylidino)-pyrimidynylthio]acetic acid (WY-14,643) (0.1\%) was added to the food, and the mice were fed for 1 week. For Yin Zhi Huang or Yin Chin treatments, appropriately matched groups of mice were gavaged once daily for 3 days with a Yin Zhi Huang or Yin Chin decoction $(10 \mathrm{ml} / \mathrm{kg} /$ day $)$. For 6,7-dimethylesculetin (Indofine Chemical Company, Hillsborough, New Jersey, USA) treatment, the compound was first dissolved in a small volume of DMSO and then mixed well with corn oil. Groups of WT and CAR null mice were injected i.p. twice daily for 3 days with vehicle control or 6,7-dimethylesculetin (100 $\mathrm{mg} / \mathrm{kg}$ ). On the fourth day, the animals were intravenously infused with bilirubin solution $(10 \mathrm{mg} / \mathrm{kg})$ via the tail vein. After 1 hour, blood was collected and total serum bilirubin was determined as described (24). Livers were removed and total bilirubin from liver was measured by diazotization with $p$-iodoaniline as described (26). Bilirubin (Sigma-Aldrich, St. Louis, Missouri, USA) was first dissolved in $0.1 \mathrm{~N} \mathrm{NaOH}$, then adjusted to $\mathrm{pH} 8.5-9.5$ with $1 \mathrm{~N} \mathrm{HCl}$, and finally diluted with isotonic saline to a concentration of $3 \mathrm{mg} / \mathrm{ml}$. The solution was always freshly prepared under lowlight conditions.

Northern bybridization. Total liver RNA was prepared using Trizol reagent (Invitrogen, Rockville, Maryland, USA) according to the manufacturer's instructions. Equivalent amounts of RNA from three to four mice were pooled, and $15 \mu \mathrm{g}$ of each sample were used for Northern blot analysis. A cytochrome P450 1A1 (CYP1A1) cDNA probe was generated by RT-PCR from mouse liver RNA. The primers used were $5^{\prime}$-AGATACCTGGGCCTCAGAGAACT- $3^{\prime}$ and $5^{\prime}$-CAGTCCATAATACAAAGCTC- $3^{\prime}$. All the other murine cDNA probes were generated as previously described $(23,24)$. In all cases, a single blot was serially hybridized with the various probes, with $\beta$-actin serving as a control for equivalent loading.

Primary hepatocyte culture. Mouse primary hepatocytes were prepared and maintained in William's E medium (Invitrogen), supplemented with $10 \mu \mathrm{g} / \mathrm{ml}$ insulin (Sigma-Aldrich) and $10^{-7} \mathrm{M}$ triamcinolone acetonide as described (27). Cells were treated with different concentrations of $4^{\prime}$-hydroxyacetophenone (Sigma-Aldrich) and 6,7-dimethylesculetin (ICC) dissolved in DMSO for 24 hours. Total RNA was isolated using the Qiagen RNeasy minikit (Qiagen, Valencia, California, USA) according to the manufacturer's instructions, and $15 \mu \mathrm{g}$ RNA were used for Northern blot analysis. For protein analysis, cells were incubated with either solvent or $50 \mu \mathrm{M}$ 6,7-dimethylesculetin for 3 hours. Fifty micrograms of nuclear extract or 50 $\mu \mathrm{g}$ of cell total protein were fractionated by PAGE and immunoblotted; hCAR was then detected with a monoclonal antibody to the c-Myc epitope tag (Roche Molecular Biochemicals, Indianapolis, Indiana, USA).

\section{Results}

CAR coordinately regulates the bilirubin clearance pathway. In addition to its function as a xenobiotic receptor, CAR responds to elevated levels of bilirubin by increasing the hepatic clearance of this toxic endobiotic (24). Several other ligand-dependent transcriptional regulators have also been shown to regulate liver responses to both xenobiotics and endobiotics, including the pregnane $X$ receptor (PXR), PPAR $\alpha$, and the aryl hydrocarbon receptor (AhR). Several lines of evidence suggest potential roles for these proteins in bilirubin clearance. Thus, the PPAR $\alpha$ ligand clofibrate has been reported to induce UGT1A1 expression (28) and to decrease circulating bilirubin levels in humans $(29,30)$. PXR has been reported to activate expression of components of the bilirubin clearance pathway, including UGT1A1 (31) and MRP2 (32). AhR, which can be activated by bilirubin $(33,34)$, has also recently been reported to activate the expression of UGT1A1 (35). Hydroxylation of bilirubin by CYP1A1, another well-known AhR target, may also contribute to bilirubin detoxification (36).

To compare the roles of these four receptors in bilirubin metabolism, groups of mice were pretreated with specific activators of each protein for 3 days, and their ability to remove bilirubin from the circulation was examined using an acute clearance assay (24). In this assay, a bolus dose of bilirubin is administered i.v., and measuring residual circulating levels after 1 hour assesses the rate of clearance. TCPOBOP significantly increased clearance as expected (24), but little or no effect was observed with the PXR activator PCN, the PPAR $\alpha$ activator WY-14,643, or the AhR activator 
a

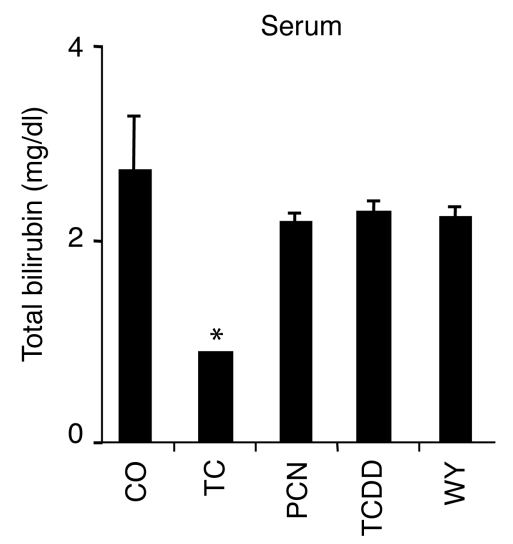

b

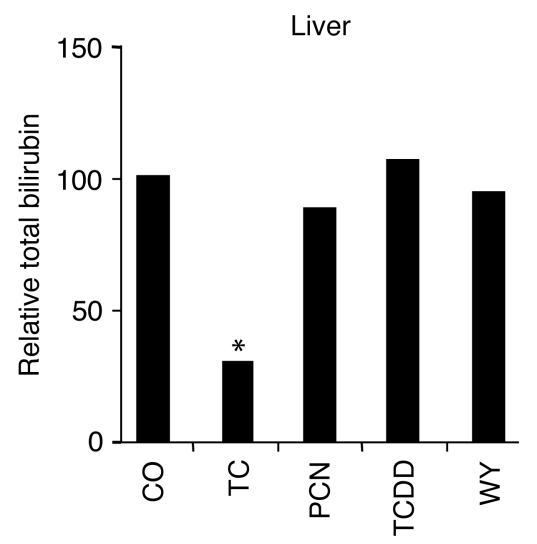

C

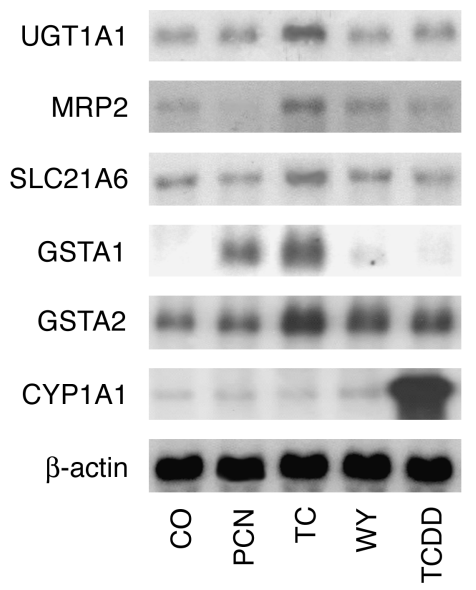

Figure 1

CAR coordinately regulates the bilirubin clearance pathway. (a) Mice were pretreated with different compounds (CO: corn oil control, TC: TCPOBOP, PCN, TCDD, and WY: WY-14,643) as indicated for 3 days. A single dose of bilirubin (10 mg/ $\mathrm{kg}$ ) was intravenously injected via the tail vein; total bilirubin was determined 60 minutes after the injection. ${ }^{*} P<0.01$ relative to the control mice. (b) Total liver bilirubin from the same mice was determined. The average value of total bilirubin from each treatment is presented as a percentage relative to the value from the control treatment. ${ }^{*} P<0.01$ relative to the control mice. (c) Total liver RNA was prepared from the same mice used in (a) and (b), and equivalent amounts of RNA from three individuals were pooled. Fifteen micrograms of each RNA sample were used for Northern hybridization with different probes as indicated.

TCDD (Figure 1a). Total hepatic bilirubin was also measured to determine whether the decrease in serum bilirubin is due to increased hepatic accumulation or actual clearance from the liver. As expected from the activation of multiple components of the clearance pathway by CAR (24), hepatic bilirubin was much lower in TCPOBOP-treated mice (Figure 1b). In contrast, PXR, PPAR $\alpha$ or AhR activation did not significantly affect hepatic bilirubin levels.

To investigate the basis for the differential responses, the expression of five genes encoding components of the bilirubin clearance pathway plus CYP1A1 was examined. TCPOBOP induced expression of all five clearance genes as expected, but PCN, WY-14,643, and TCDD had more restricted and moderate effects (Figure 1c). ТСРОВOP increased UGT1A1 mRNA levels fourfold, for example, whereas the response to PCN and TCDD was less than twofold, and WY-14,643 had no apparent effect. The limited responses are not due to lack of effects of the activators, since appropriate target genes were induced in each case. Thus, PXR activation by PCN strongly induced glutathione transferase GSTA1 expression, and TCDD-activated AhR strongly induced CYP1A1 expression. Similarly, PPAR $\alpha$ activa-

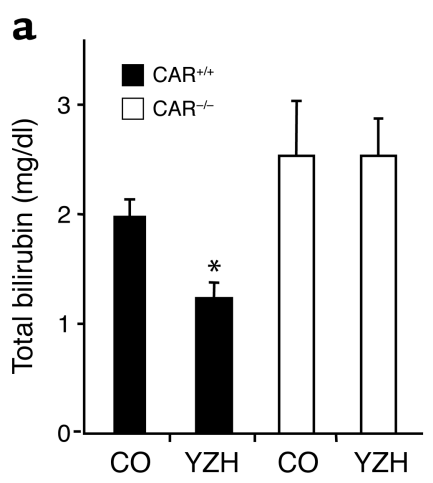

b

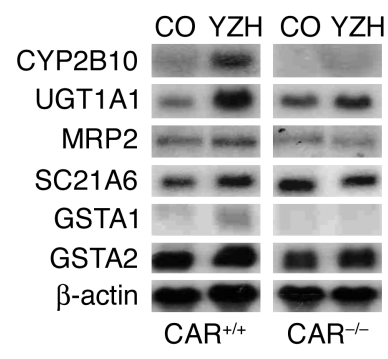

C

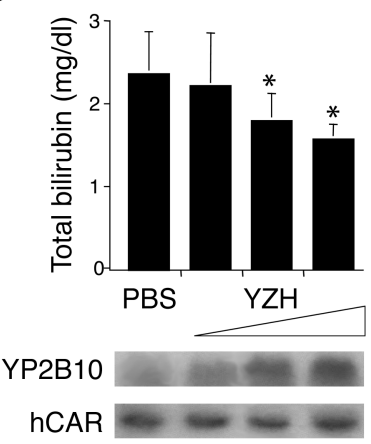

Figure 2

Yin Zhi Huang treatment stimulates bilirubin clearance in WT, but not CAR, knockout mice. (a) Groups of four WT or CAR ${ }^{-/-}$mice were gavaged with either saline (PBS), control (CO), or Yin Zhi Huang (YZH) decoction (10 ml/kg/day) for 3 days. On the fourth day, mice were subjected to an acute bilirubin clearance assay, and total serum bilirubin was determined as described. ${ }^{*} P<0.05$ relative to the control mice. (b) Total liver RNA was prepared from the animals as in (a). Equivalent amounts of RNA were pooled from four individuals, and $15 \mu \mathrm{g}$ of each RNA sample was used for Northern hybridization with different probes as indicated. (c) Four groups of humanized CAR mice were treated with either PBS or increasing concentrations of $\mathrm{YZH}(2.5,5$ or $10 \mathrm{ml} / \mathrm{kg} /$ day) for 3 days. An acute bilirubin clearance assay, as well as Northern hybridization with CYP2B10 and human CAR probes, was carried out. 


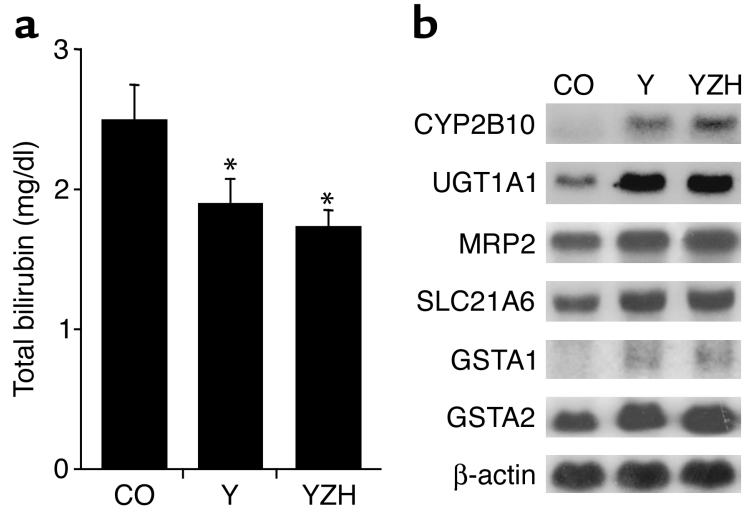

Figure 3

Human CAR can mediate response to Yin Zhi Huang. (a) Humanized $C A R$ mice between 8 and 10 weeks of age were separated into three groups and received either PBS (CO), Yin Chin (Y), or Yin Zhi Huang ( $\mathrm{YZH}$ ) decoction via gavage for 3 days $(10 \mathrm{ml} / \mathrm{kg} /$ day). On the fourth day, acute bilirubin clearance assay and total bilirubin measurement were performed as described. ${ }^{*} P<0.05$ relative to control. (b) Total liver RNA from the same animals was prepared and used for Northern hybridization with different probes as indicated.

tion by WY-16,643 increased liver cell proliferation in these mice as expected (data not shown). Although it remains possible that other receptors contribute to bilirubin metabolism in at least some circumstances, these results identify CAR as a particularly important regulator of this process.

Yin Zhi Huang stimulation of bilirubin clearance is dependent on CAR. The primary role of CAR in bilirubin metabolism is consistent with earlier studies showing positive effects of phenobarbital on jaundice $(37,38)$. To test the possibility that CAR also mediates the effect of Yin Zhi Huang on bilirubin clearance, both WT and CAR knockout mice were pretreated with Yin Zhi Huang or saline control by oral gavage for 3 days. On the fourth day, mice were subjected to an acute bilirubin clearance assay. In agreement with previous results (19), Yin Zhi Huang treatment of WT mice increased the clearance rate. However, this effect was completely absent in CAR knockout animals, demonstrating that CAR is required to mediate the effect of this herbal decoction on bilirubin clearance (Figure 2a).

To examine the mechanism of enhanced bilirubin clearance by Yin Zhi Huang, hepatic expression of the primary CAR target gene CYP2B10 and the five components of the clearance pathway were examined. In the WT mice, all the genes showed at least some response, with particularly strong induction of CYP2B10 and UGT1A1 (Figure 2b). Except for UGT1A1, which showed a modest residual response, these inductions were absent in the CAR null animals. The weak induction of UGT1A1 in CAR knockout animals may indicate some effect of Yin Zhi Huang on PXR or other receptors.

The effect of Yin Zhi Huang on bilirubin clearance decreases with decreasing doses (Figure 2c). Additional support for the role of CAR in this response is provided by the observation that a similar dose dependence is observed for the increased rate of bilirubin clearance and the induction of CYP2B10 mRNA. Yin Zhi Huang treatment did not affect CAR expression.<smiles>COc1cc2ccc(=O)oc2cc1OC</smiles>

6,7-Dimethylesculetin<smiles>COc1ccc(O)cc1</smiles>

4'-Hydroxyacetophenone

b

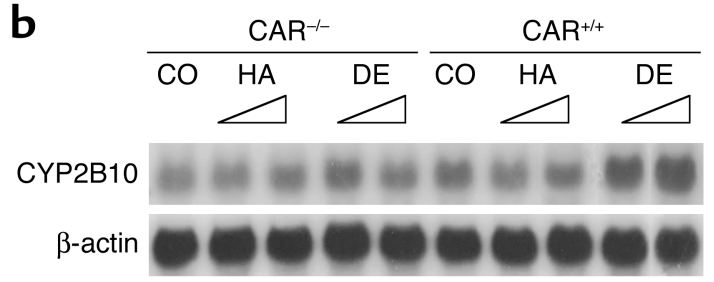

C

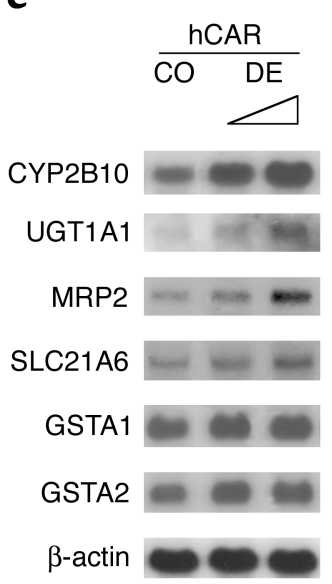

d

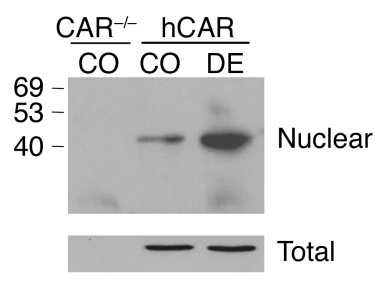

Figure 4

6,7-Dimethylesculetin activates CAR in primary hepatocytes. (a) Structures of 6,7-dimethylesculetin and 4'-hydroxyacetophenone. (b) Primary hepatocytes from either WT (+/+) or CAR knockout animals (-/-) were cultured in William's E medium (Invitrogen) and incubated with solvent $(\mathrm{CO})$ or increasing concentrations of $4^{\prime}$-hydroxyacetophenone (HA; $10 \mu \mathrm{M}$, or $\left.50 \mu \mathrm{M}\right)$, or 6,7-dimethylesculetin (DE; $10 \mu \mathrm{M}$, or $50 \mu \mathrm{M}$ ) for 24 hours. Total cell RNA was prepared, and $15 \mu \mathrm{g}$ of each RNA sample were used for Northern hybridization as indicated. (c) Primary hepatocytes were isolated from humanized CAR transgenic mice and cultured in the presence of either solvent or $10 \mu \mathrm{M}$ or $50 \mu \mathrm{M}$ 6,7-dimethylesculetin for 24 hours. Fifteen micrograms of total RNA from cells were analyzed by Northern hybridization. (d) Primary hepatocytes were isolated from either CAR knockout or humanized CAR transgenic mice and cultured in the presence of either solvent, or $50 \mu \mathrm{M}$ 6,7-dimethylesculetin for 3 hours. Fifty micrograms of nuclear extract protein (upper panel) or $50 \mu \mathrm{g}$ of cell total protein (lower panel) from each treatment were fractionated by SDS-PAGE and immunoblotted with an anti c-Myc antibody that recognizes the $N$-terminal epitope tag on the human CAR expressed in these animals. 
a

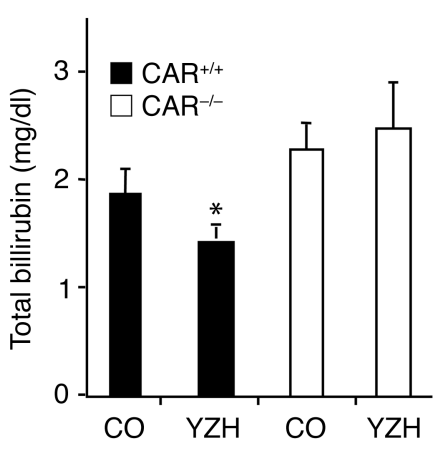

b CYP2B10
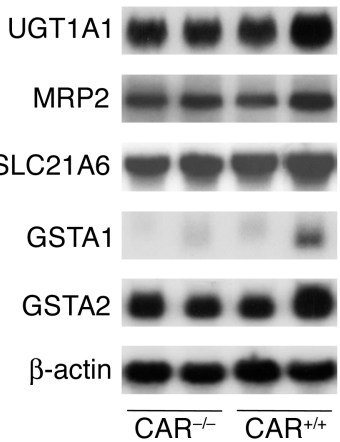

Figure 5

6,7-Dimethylesculetin accelerates bilirubin clearance in vivo. (a) Groups of five to six WT or $\mathrm{CAR}^{-/-}$mice were injected with either vehicle control (CO) or 6,7-dimethylesculetin (DE) $(100 \mathrm{mg} / \mathrm{kg})$ twice for 3 days. On the fourth day, mice were subjected to an acute bilirubin clearance assay. ${ }^{*} P<0.05$ relative to WT control. (b) Total liver RNA was prepared from the animals and equivalent amounts of RNA were pooled from individual mice; $15 \mu \mathrm{g}$ of each RNA sample were used for Northern analysis with probes as indicated.

Because murine and human CAR can show quite different responses to different activators, it was important to test whether human CAR can also mediate effects of Yin Zhi Huang on bilirubin clearance. These studies used previously described humanized mice expressing human instead of murine CAR in the liver $(24,25)$. These humanized mice do not respond to TCPOBOP, which is specific for the murine CAR, but they do respond to the general CAR activator phenobarbital and the specific human CAR agonist 6-(4-chlorophenyl)imidazo[2,1-b][1,3]thiazole-5carbaldehyde O-(3,4-dichlorobenzyl)oxime (39). Three groups of humanized mice received either saline control, Yin Zhi Huang, or Yin Chin alone, which is considered to be the major active herb in the Yin Zhi Huang mixture. Although the response was somewhat less than that of the WT mice, as previously observed with phenobarbital activation of human CAR (24), both Yin Zhi Huang and Yin Chin significantly enhanced the clearance of the exogenously infused bilirubin (Figure 3a). As expected from the results with the WT mice, expression of a number of CAR target genes was also increased by Yin Zhi Huang and Yin Chin in the humanized mice (Figure $3 b$ ).

6,7-Dimethylesculetin activates both mouse and human CAR. These results indicate that Yin Chin contains an agent or agents that can activate both mouse and human CAR. Among a number of compounds present in Yin Chin are the coumarin 6,7-dimethylesculetin (scoparone) and 4'-hydroxyacetophenone (Figure 4a). 6,7-Dimethylesculetin is a major constituent, comprising up to $2 \%$ of Yin Chin by dry weight and has been associated with a number of potential biologic actions (40).

In tests of the effects of the compounds in primary hepatocytes from WT mice, 6,7-dimethylesculetin treatment significantly induced CYP2B10 expression, but

CAR knockout hepatocytes showed no response (Figure 4b). 4'-Hydroxyacetophenone had no effect in either case. In primary hepatocytes from humanized $C A R$ mice, 6,7-dimethylesculetin also induced expression of CAR target genes, especially CYP2B10, UGT1A1, and MRP2; lesser responses of SLC21A6 and GSTA1 and GSTA2 were confirmed by densitometry (Figure 4c).

A variety of transient transfections with full-length receptors or mammalian two-hybrid assays for coactivator recruitment failed to provide evidence that either these compounds or several other candidates tested function as direct CAR agonist ligands (data not shown). In hepatocytes, however, CAR can also be activated by an indirect mechanism based on a specifically induced translocation from the cytoplasm to the nucleus (41). The effect of 6,7-dimethylesculetin on the levels of CAR present in the nuclei of primary hepatocytes from humanized CAR mice was examined by Western blotting. A low basal level of nuclear CAR was detected in the untreated hepatocytes, which may account for the increased basal expression of CYP2B10 in the cultured cells relative to the liver. As expected, however, 6,7-dimethylesculetin treatment significantly increased the levels of CAR in the nucleus but did not affect the total amount of human CAR (Figure 4d).

To investigate the effect of 6,7-dimethylesculetin on bilirubin clearance in vivo, we treated the WT and CAR null mice twice daily with 6,7-dimethylesculetin or a vehicle control for 3 days. The effects of the pure compound were quite similar to those of the Yin Chin extract, with an increase in both bilirubin clearance and expression of CAR target genes (Figure 5). Overall, we concluded that 6,7-dimethylesculetin is a specific CAR activator that is likely to contribute to the ability of Yin Zhi Huang to increase bilirubin clearance.

\section{Discussion}

Both previous results (24) and those described here demonstrate that CAR is a key regulator of bilirubin clearance. Under ordinary circumstances, CAR is sequestered in the hepatocyte cytoplasm and thus does not affect bilirubin levels. In response to elevated bilirubin levels, however, CAR activates expression of multiple components of the bilirubin clearance pathway, resulting in an increased rate of clearance.

Several other receptors have been reported to induce expression of the primary bilirubin-conjugating enzyme UGT1A1, including the nuclear receptors PXR (31) and PPAR $\alpha(28)$ and the per-arnt-sim domain protein AhR (35). The potential response of this important detoxifying enzyme to diverse signals is a reflection of the complexity of the functional interrelationships of these regulators. For example, numerous studies indicate that both CAR and PXR can induce expression of a number of genes encoding various components of drug metabolism pathways (42-44). Although this can result in common regulatory effects, the regulation of common target genes does not mean that the distinct receptors have the same physiologic effects. Thus, the results 
described here indicate that acute activation of CAR has a particularly significant impact on the overall bilirubin clearance rate, although it remains possible that important functions for the other receptors would be observed under other circumstances.

The results described here also demonstrate that CAR mediates the effects of the Chinese traditional medicine Yin Zhi Huang on bilirubin clearance. In Chinese and other traditional medicines, disease is thought to be a consequence of imbalances in the body, and it is often considered necessary to incorporate multiple components into a therapeutic approach to restore balance to different processes. Yin Zhi Huang is a typical example of such a combination. Because other combinations of Yin Chin with quite different components are also used in Chinese and other Asian traditional medicines with apparently similar effects, Yin Chin is thought to be the primary active agent in this mixture. Consistent with this, both Yin Chin and 6,7-dimethylesculetin are sufficient to induce both bilirubin clearance and CAR target gene expression, and we conclude that 6,7-dimethylesculetin is an active component of Yin Zhi Huang and other Yin Chin-containing herbal medicines that contributes to their biological effects. Particularly because previous studies indicate that extracts of the other plants in Yin Zhi Huang can increase GST or UGT1A1 activity (45), it is possible that other components also contribute.

It is intriguing that Artemisia species related to Yin Chin (e.g., wormwood, absinthe, mugwort, and tarragon) have been used in traditional medicines from many cultures for a variety of indications, including liver ailments, and a number of studies in animal models indicate hepatoprotective effects for various Artemisia extracts $(46,47)$. These effects have been attributed to antioxidant or other properties of the components of these extracts, but it is an interesting possibility that CAR activation is a contributing factor.

In summary, we have demonstrated the particular importance of CAR in regulating bilirubin clearance, demonstrated that this nuclear receptor mediates the effect of the natural herbal medicine Yin Zhi Huang on this process, and identified a specific active compound present in this mixture. CAR thus joins farnesoid X receptor (FXR) $(48)$ and PXR $(49,50)$ as recently identified targets of significant biological effects of herbal medicines. We believe that these and other nuclear receptors with important metabolic regulatory functions will be found to mediate activities of other natural products. Based on the recent identification of a specific human CAR agonist (39), we also anticipate that more modern approaches to drug design based on CAR may yield new treatments for neonatal and other forms of jaundice.

\section{Acknowledgments}

We thank Steve S. Chua for generating the humanized CAR mice and Amethyst C. Kurbegov for preliminary results. This work was supported by NIH grant DK46546.
1. Dennery, P.A., Seidman, D.S., and Stevenson, D.K. 2001. Neonatal hyperbilirubinemia. N. Engl. J. Med. 344:581-590.

2. Arias, I.M., Gartner, L.M., Cohen, M., Ezzer, J.B., and Levi, A.J. 1969. Chronic nonhemolytic unconjugated hyperbilirubinemia with glucuronyl transferase deficiency. Clinical, biochemical, pharmacologic and genetic evidence for heterogeneity. Am. J. Med. 47:395-409.

3. Kamisako, T., et al. 2000. Recent advances in bilirubin metabolism research: the molecular mechanism of hepatocyte bilirubin transport and its clinical relevance. J. Gastroenterol. 35:659-664.

4. Sima, P., Mala, J., Miler, I., Hodr, R., and Truxova, E. 1980. The suppressive effect of continuous infusion of bilirubin on the immune response in mice. Folia Microbiol. (Praha) 25:483-490.

5. Scott-Conner, C.E., and Grogan, J.B. 1994. The pathophysiology of biliary obstruction and its effect on phagocytic and immune function. J. Surg. Res. 57:316-336.

6. Jansen, P.L., Bosma, P.J., and Chowdhury, J.R. 1995. Molecular biology of bilirubin metabolism. Prog. Liver Dis. 13:125-150.

7. Cui, Y., Konig, J., Leier, I., Buchholz, U., and Keppler, D. 2001. Hepatic uptake of bilirubin and its conjugates by the human organic anion transporter SLC21A6. J. Biol. Chem. 276:9626-9630.

8. Dennery, P.A. 2002. Pharmacological interventions for the treatment of neonatal jaundice. Semin. Neonatol. 7:111-119.

9. Catz, C., and Yaffe, S.J. 1962. Pharmacological modification of bilirubin conjugation in the newborn. Am. J. Dis. Child. 104:516-517.

10. Ritter, J.K., et al. 1999. Expression and inducibility of the human bilirubin UDP-glucuronosyltransferase UGT1A1 in liver and cultured primary hepatocytes: evidence for both genetic and environmental influences. Hepatology. 30:476-484.

11. Yaffe, S.J., Levy, G., Matsuzawa, T., and Baliah, T. 1966. Enhancement of glucuronide-conjugating capacity in a hyperbilirubinemic infant due to apparent enzyme induction by phenobarbital. N. Engl. J. Med. 275:1461-1466.

12. Valaes, T., Kipouros, K., Petmezaki, S., Solman, M., and Doxiadis, S.A 1980. Effectiveness and safety of prenatal phenobarbital for the prevention of neonatal jaundice. Pediatr. Res. 14:947-952.

13. Wallin, A., and Boreus, L.O. 1984. Phenobarbital prophylaxis for hyperbilirubinemia in preterm infants. A controlled study of bilirubin disappearance and infant behavior. Acta Paediatr. Scand. 73:488-497.

14. Fok, T.F. 2001. Neonatal jaundice - traditional Chinese medicine approach. J. Perinatol. 21 Suppl 1:S98-S100; discussion S104-S107.

15. Yang, S.H., and Lu, C.F. 1984. Effects of decoction of Artemisia, Rheum, Gardenia, Coptidis and Rhizoma on neonatal jaundice in Chinese newborn infants. J. Chin. Child Med. 25:144-148.

16. Chen, Z.L., and Guan, W.H. 1985. Approach to the effect and indication of Yin Zhi Huang to treat neonatal jaundice. J. Clin. Pediatr. 3:302-303.

17. Dong, Y.S., Huang, Z.H., and Wu, L.F. 1992. Treatment of infantile hepatitis syndrome with injection of yin zhi huang [In Chinese]. Zhongguo Zhong Xi Yi Jie He Za Zhi. 12:26-27, 25-26.

18. Yin, J., Wennberg, R.P., Xia, Y.C., Liu, J.W., and Zhou, H.Z. 1991. Effect of a traditional Chinese medicine, yin zhi huang, on bilirubin clearance and conjugation. Dev. Pharmacol. Ther. 16:59-64.

19. Yin, J., Miller, M., and Wennberg, R.P. 1991. Induction of hepatic bilirubin-metabolizing enzymes by the traditional Chinese medicine yin zhi huang. Dev. Pharmacol. Ther. 16:176-184.

20. Honkakoski, P., Zelko, I., Sueyoshi, T., and Negishi, M. 1998. The nuclear orphan receptor CAR-retinoid $\mathrm{X}$ receptor heterodimer activates the phenobarbital-responsive enhancer module of the CYP2B gene. Mol. Cell. Biol. 18:5652-5658.

21. Sueyoshi, T., Kawamoto, T., Zelko, I., Honkakoski, P., and Negishi, M. 1999. The repressed nuclear receptor CAR responds to phenobarbital in activating the human CYP2B6 gene. J. Biol. Chem. 274:6043-6046.

22. Tzameli, I., Pissios, P., Schuetz, E.G., and Moore, D.D. 2000. The xenobiotic compound 1,4-Bis[2-(3,5-dichloropyridyloxy)]benzene is an agonist ligand for the nuclear receptor CAR. Mol. Cell. Biol. 20:2951-2958.

23. Wei, P., Zhang, J., Egan-Hafley, M., Liang, S., and Moore, D.D. 2000. The nuclear receptor CAR mediates specific xenobiotic induction of drug metabolism. Nature. 407:920-923.

24. Huang, W., et al. 2003. Induction of bilirubin clearance by the xenobiotic receptor CAR. Proc. Natl. Acad. Sci. U. S. A. In press.

25. Zhang, J., Huang, W., Chua, S.S., Wei, P., and Moore, D.D. 2002. Modulation of acetaminophen-induced hepatotoxicity by the xenobiotic receptor CAR. Science. 298:422-424.

26. Van Roy, F.P., Meuwissen, J.A., De Meuter, F., and Heirwegh, K.P. 1971. Determination of bilirubin in liver homogenates and serum with diazotized p-iodoaniline. Clin. Chim. Acta. 31:109-118.

27. Kocarek, T.A., Kraniak, J.M., and Reddy, A.B. 1998. Regulation of rat hepatic cytochrome P450 expression by sterol biosynthesis inhibition: inhibitors of squalene synthase are potent inducers of CYP2B expression in primary cultured rat hepatocytes and rat liver. Mol. Pharmacol. 54:474-484. 
28. Jemnitz, K., Lengyel, G., and Vereczkey, L. 2002. In vitro induction of bilirubin conjugation in primary rat hepatocyte culture. Biochem. Biophys. Res. Commun. 291:29-33.

29. Kutz, K., Kandler, H., Gugler, R., and Fevery, J. 1984. Effect of clofibrate on the metabolism of bilirubin, bromosulphophthalein and indocyanine green and on the biliary lipid composition in Gilbert's syndrome. Clin. Sci. (Lond.). 66:389-397.

30. Gabilan, J.C., Benattar, C., and Lindenbaum, A. 1990. Clofibrate treatment of neonatal jaundice. Pediatrics. 86:647-648.

31. Xie, W., et al. 2003. Activation of UDP-Glucuronosyltransferases by nuclear receptors PXR and CAR and its implication in hormonal homeostasis. Proc. Natl. Acad. Sci. U. S. A. In press.

32. Kast, H.R., et al. 2001. Regulation of multidrug resistance-associated protein 2 (MRP2;ABCC2) by the nuclear receptors PXR, FXR, and CAR. J. Biol. Chem. 12:12

33. Sinal, C.J., and Bend, J.R. 1997. Aryl hydrocarbon receptor-dependent induction of cyp $1 \mathrm{a} 1$ by bilirubin in mouse hepatoma hepa $1 \mathrm{c} 1 \mathrm{c} 7 \mathrm{cells}$. Mol. Pharmacol. 52:590-599.

34. Phelan, D., Winter, G.M., Rogers, W.J., Lam, J.C., and Denison, M.S. 1998. Activation of the Ah receptor signal transduction pathway by bilirubin and biliverdin. Arch. Biochem. Biophys. 357:155-163.

35. Yueh, M.F., Huang, Y.H., Chen, S., Nguyen, N., and Tukey, R.H. 2003. Involvement of the xenobiotic response element (XRE) in Ah-receptor mediated induction of human UDP-glucuronosyltransferase 1A1. J. Biol. Chem. 278:15001-15006.

36. De Matteis, F., Dawson, S.J., Boobis, A.R., and Comoglio, A. 1991. Inducible bilirubin-degrading system of rat liver microsomes: role of cytochrome P450IA1. Mol. Pharmacol. 40:686-691.

37. Stern, L., Khanna, N.N., Levy, G., and Yaffe, S.J. 1970. Effect of phenobarbital on hyperbilirubinemia and glucuronide formation in newborns. Am. J. Dis. Child. 120:26-31.

38. Yeung, C.Y., Tam, L.S., Chan, A., and Lee, K.H. 1971. Phenobarbitone prophylaxis for neonatal hyperbilirubinemia. Pediatrics. 48:372-376.

39. Maglich, J.M., et al. 2003. Identification of a novel human CAR ago- nist and its use in the identification of CAR target genes. J. Biol. Chem 278: $17277-17283$

40. Hoult, J.R., and Paya, M. 1996. Pharmacological and biochemical actions of simple coumarins: natural products with therapeutic potential. Gen. Pharmacol. 27:713-722.

41. Kawamoto, T., et al. 1999. Phenobarbital-responsive nuclear translocation of the receptor CAR in induction of the CYP2B gene. Mol. Cell. Biol. 19:6318-6322.

42. Xie, W., et al. 2000. Reciprocal activation of xenobiotic response genes by nuclear receptors SXR/PXR and CAR. Genes Dev. 14:3014-3023.

43. Maglich, J.M., et al. 2002. Nuclear pregnane $\mathrm{x}$ receptor and constitutive androstane receptor regulate overlapping but distinct sets of genes involved in xenobiotic detoxification. Mol. Pharmacol. 62:638-646.

44. Wei, P., Zhang, J., Dohan, D., Han, Y., and Moore, D.D. 2002. Specific and overlapping functions of the nuclear hormone receptors CAR and PXR in xenobiotic response. Pharmacogenomics J. 2:117-126.

45. Yin, J., Wennberg, R.P., and Miller, M. 1993. Induction of hepatic bilirubin and drug metabolizing enzymes by individual herbs present in the traditional Chinese medicine, yin zhi huang. Dev. Pharmacol. Ther. 20:186-194.

46. Aniya, Y., et al. 2000. Antioxidant and hepatoprotective actions of the medicinal herb Artemisia campestris from the Okinawa Islands. Biol. Pharm. Bull. 23:309-312.

47. Park, E.J., et al. 2000. The ethanol-soluble part of a hot-water extract from Artemisia iwayomogi inhibits liver fibrosis induced by carbon tetrachloride in rats. J. Pharm. Pharmacol. 52:875-881.

48. Urizar, N.L., et al. 2002. A natural product that lowers cholesterol as an antagonist ligand for FXR. Science. 296:1703-1706.

49. Moore, L.B., et al. 2000. St. John's wort induces hepatic drug metabolism through activation of the pregnane X receptor. Proc. Natl. Acad. Sci. U. S. A. 97:7500-7502.

50. Wentworth, J.M., Agostini, M., Love, J., Schwabe, J.W., and Chatterjee, V.K. 2000. St. John's wort, a herbal antidepressant, activates the steroid X receptor. J. Endocrinol. 166:R11-R16. 